\title{
Lernen verändert sich - auch für Ärztinnen und Ärzte
}

\begin{abstract}
Neue Lernformen sind auf dem Vormarsch und prägen die ärztliche Aus-, Weiter- und Fortbildung zusehends und nachhaltig. Diesen Eindruck vermittelte ein Symposium zum Thema «Blended Learning», das im Februar dieses Jahres im Zürcher UniversitätsSpital durchgeführt wurde.
\end{abstract}

\author{
Bruno Kesseli (Text und Bilder) \\ Dr. med. et lic. phil., Chefredaktor
}

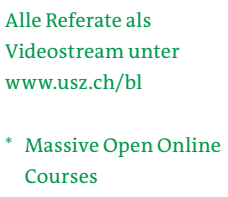

Noch sind Begriffe wie «Blended Learning» oder «Flipped Classroom» nicht in aller Munde, wie Moderator Jan Breckwoldt vom Studiendekanat der Medizinischen Fakultät der Universität Zürich bei seiner Begrüssungsansprache wohl richtig vermutete. Jürg Hodler, ärztlicher Direktor des UniversitätsSpitals Zürich (USZ), bestätigte scherzhaft, der Neologismus habe in seinem Umfeld zunächst die Frage evoziert, ob das Universitätsspital nun auch noch in die Whiskyproduktion einsteigen wolle. Im Übrigen bekannte er sich vorbehaltlos $\mathrm{zu}$ einer Aussage von Sir William Osler aus dem Jahr 1903: «The work of an institution in which there is no teaching is rarely first class.» Ein Zitat, das wiederum den bekennenden Osler-Anhänger Werner Bauer freute, musste der Präsident des Schweizerischen Instituts für ärztliche Weiter- und Fortbildung (SIWF) doch in seinem Referat aus Zeitgründen und entsprechend schweren Herzens darauf verzichten, den kanadischen Übervater der Internisten zu Wort kommen zu lassen. Als der ärztlichen Bildung verpflichteter, innovativer Geist hätte Osler die Möglichkeiten des Blended Learning wohl positiv bewertet. Auch Jürg Hodler und Werner Bauer wiesen vor dem Hintergrund der - nicht zuletzt in finanzieller Hinsicht - schwierigen Rahmenbedingungen, unter denen die ärztliche Weiterbildung

\section{Symposium Blended Learning}

Les nouvelles formes d'apprentissage ont le vent en poupe et influencent aussi de plus en plus durablement la formation médicale prégraduée, postgraduée et continue. C'est du moins l'impression qui demeure suite au symposium sur le "blended learning" qui s'est tenu en février dernier à I'hôpital universitaire de Zurich. Le "blended learning" combine l'apprentissage en ligne indépendamment du lieu et du temps avec des méthodes d'apprentissage plus traditionnelles, principalement des cours en présence, dans le but d'obtenir une solution de formation optimale qui utilise les avantages offerts par les différents outils à disposition tout en limitant leurs inconvénients. gegenwärtig zu gewährleisten ist, auf das Potential dieser neuen Form des Lernens hin.

\section{Ziel: optimales Lernarrangement}

Doch was ist mit Blended Learning, für das auch der deutsche Begriff «Integriertes Lernen» verwendet wird, überhaupt gemeint? Im Allgemeinen wird darunter die didaktisch sinnvolle Kombination des weitgehend zeit- und ortsunabhängigen E-Learning mit traditionellen Lernmethoden verstanden, die meist im Rahmen von Präsenzveranstaltungen zum Zug kommen. Ziel ist ein optimales Lernarrangement, das die Stärken der verschiedenen zur Verfügung stehenden Instrumente optimal nutzt und deren Schwächen minimiert.

Das ist kein alter Wein in neuen Schläuchen, denn die Fortschritte auf dem Gebiet der Informationstechnologien eröffnen im Bereich des Lernens eine Vielzahl von Möglichkeiten, die es früher schlicht nicht gab. Ein Beispiel sind die sogenannten MOOCS*. Es handelt sich dabei um offene Online-Lehrveranstaltungen mit potentiell enormen Teilnehmerzahlen, in denen die Wissensvermittlung via Internet über verschiedene, teilweise interaktiv angelegte Instrumente wie Videos, Lesematerial, Aufgabenstellungen oder Foren erfolgt. Ein direkter Austausch ist sowohl zwischen den Studierenden, beispielsweise in Foren oder Arbeitsgruppen, aber auch mit den Dozierenden möglich.

\section{Das umgedrehte Klassenzimmer}

Organisator Hans Ulrich Bucher, ehemaliger Direktor der Klinik für Neonatologie und Delegierter für ärztliche Weiterbildung des UniversitätsSpitals Zürich, hatte Referenten mit sehr unterschiedlichen Zugängen zum Thema des Symposiums eingeladen. Als ausgewiesene Expertin auf dem Gebiet des Blended Learning kann 
Martina Kadmon gelten. Die Chirurgin und Professorin für Medizinische Ausbildung an der Universität Oldenburg machte in ihrem - durch interaktive Einschübe aufgelockerten - Referat deutlich, dass es bei dieser Form des Lernens nicht darum geht, Präsenzveranstaltungen zu reduzieren oder gar aufzugeben. Die Chancen des Blended Learning bestünden unter anderem darin, dass dieses Konzept zumindest Teil-Antworten bieten könne in Bereichen wie den steigenden Weiterbildungskosten oder der Flexibilisierung von Arbeitsund Weiterbildungszeiten.

Aus dem Publikum wurde von eigenen Erfahrungen berichtet. Offensichtlich ist es in verschiedenen Häusern bereits Usus, dass für die Zulassung zu bestimmten praktisch ausgerichteten Fortbildungsveranstaltungen oder praktischen ärztlichen Tätigkeiten - etwa zu Reanimationskursen oder laparoskopischen Operationen - online erworbene Fähigkeiten nachgewiesen werden müssen und eine entsprechende Prüfung zu bestehen ist. Die e-Lernenden eignen sich also Wissen oder bestimmte Fähigkeiten mit grossen Freiheiten in Bezug auf Zeit und Ort an und haben die Möglichkeit, Nichtverstandenes individuell zu repetieren. An den Präsenzveranstaltungen wird nicht mehr primär (Basis-)Wissen vermittelt, sondern das Gelernte angewandt, geübt und vertieft.

Diese neue Form des Lernens wird als «Flipped Classroom» bezeichnet. Wurde früher das Wissen im Klassenzimmer vermittelt und später zu Hause geübt und vertieft, ist es nun umgekehrt. Martina Kadmon illustrierte die Vielfalt der im Rahmen von Blended Learning möglichen Lernformen und Interaktionsmöglichkeiten - auch Smartphones und Tablets sind selbstverständlich Bestandteil des Instrumentariums - mit einer Reihe von Beispielen. Virtuelle Patienten können genauso dazugehören wie Online-Simulationstrainings oder von Dozenten kommentierte audiovisuelle Präsentationen im Internet. Die Bilanz des Blended Learning ist aus ihrer Sicht klar positiv, wenngleich noch nicht sehr gut untersucht sei, wie der optimale Blend aussehe.

\section{Besser vorbereitet ans Krankenbett}

Noch konkreter wurde es in der Präsentation von Urs Haller. Der ehemalige Vorsteher des Departements für Frauenheilkunde und Direktor der Klinik für Gynäkologie des UniversitätsSpitals Zürich stellte in Form einer direkten Demonstration das E-Learning-Portal EGONE Swiss Virtual Campus vor, an dem er als Projektleiter federführend beteiligt war. Während Egone basic für Medizinstudierende in der klinischen Ausbildung konzipiert ist, deckt Egone plus die Bedürfnisse

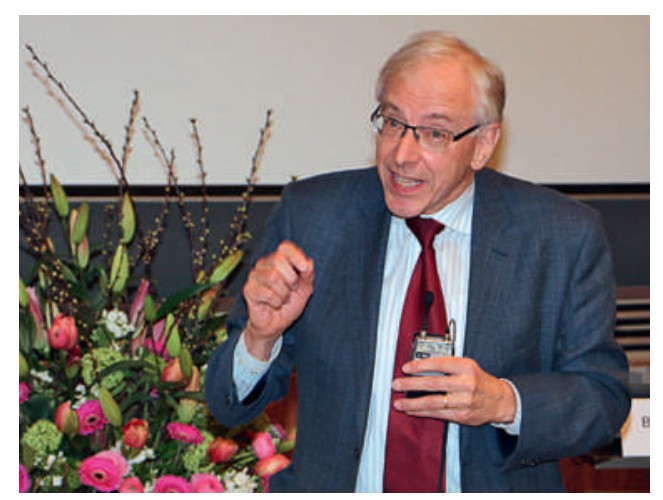

Organisator Hans Ulrich Bucher, der Delegierte für ärztliche Weiterbildung des UniversitätsSpitals Zürich (USZ).

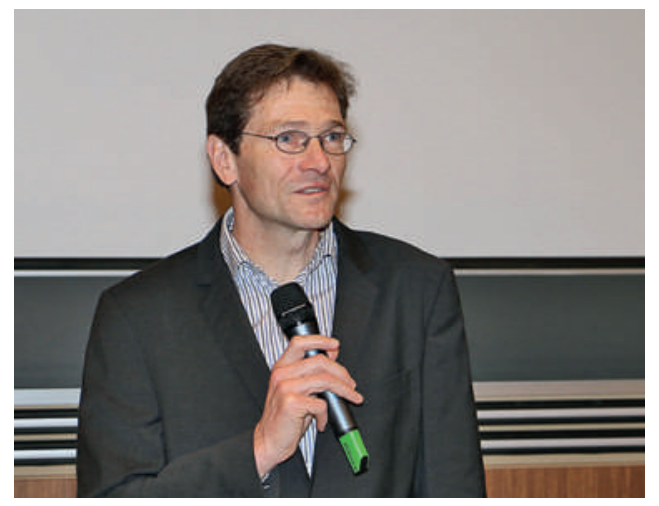

Moderator Jan Breckwoldt.

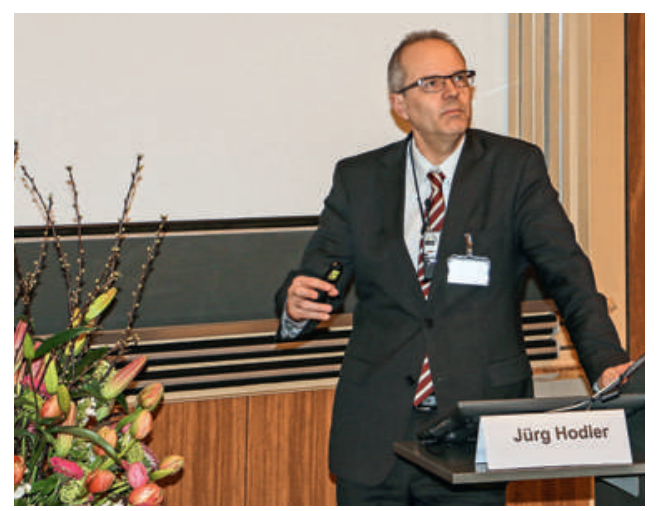

Jürg Hodler, Ärztlicher Direktor des USZ.

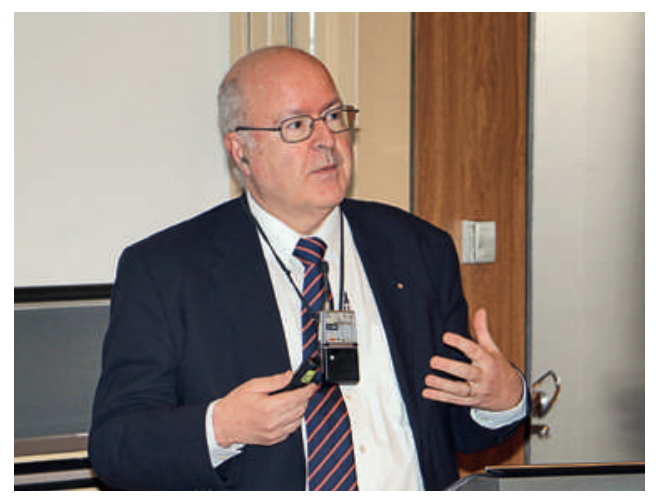

Werner Bauer, Präsident des SIWF. 
der Ärztinnen und Ärzte in Weiter- und Fortbildung ab. Das Portal bietet eine Lernumgebung, von der frühere Generationen von Aus-, Weiter- und Fortzubildenden nur träumen konnten und ermöglicht zielgerichtetes Lernen mittels eines Portfolios an systematischen, deskriptiven, explorativen und $\mathrm{PBL}^{*}$-orientierten Online-Komponenten. Aktuelle Fortbildungsveranstaltungen und interaktive Videos mit Selbstkontrolle der persönlichen Lernfortschritte sollen das Wissen der Nutzer auf dem neuestem Stand halten, zusätzliche Fortbildungs-Module die «Continuous Medical Education» gewährleisten. Gemäss Haller zeigen sich positive Auswirkungen von E-Learning-Tools beispielsweise darin, dass die Studierenden meist gut vorbereitet zum Teaching am Krankenbett erscheinen, was einen echten Austausch anstelle der früher üblichen Einwegkommunikation begünstige und interessante Diskussionen ermögliche.

Die weiteren Referate der Ethikerin Tanja Krones und von Adrian Marty, Leiter des Simulationszentrums des UniversitätsSpitals Zürich rundeten den «Blend» des Symposiums stimmig ab. Auch im Bereich der klinischen Ethik, die häufig mit Fallsituationen konfrontiert ist, haben Elemente des Blended Learning mittlerweile ihren festen Platz. Krones stellte unter anderem das E-Learning-Instrument «Reanimationsentscheide» vor, das aus einer dreiteiligen Lerneinheit und einem Abschlusstest besteht. Marty vermittelte anschaulich, wie Simulationen auch im Bereich der Medizin zusehends realistischer werden. Neben der «Physical Fidelity" braucht es dazu auch die "Conceptual Fidelity" und die «Emotional Fidelity».

\section{Fazit: Blended Learning ist mehr als ein Modetrend}

Auf dem abschliessenden, von Balthasar Eugster moderierten Podium waren sich die Referenten einig, dass das Konzept des Blended Learning nicht nur ein Modetrend ist, sondern die Aus-, Weiter- und Fortbildung nachhaltig verändern und prägen wird. Dabei wurde betont, dass der «Blend» stimmen muss und E-Learning-Projekte vor allem dann hilfreich sind, wenn sie dazu beitragen, Zeit für die Vermittlung des «klinischen Denkstils» zu gewinnen. Teaching, so wurde argumentiert, sei nach wie vor unverzichtbar, der Transfer "from Bench- to Bedside» entscheidend. Präsenzveranstaltungen, der direkte Austausch zwischen Lernendem und Lehrendem, würden auch in Zukunft ein tragender Pfeiler im Bereich von Aus-, Weiter- und Fortbildung bleiben. Und nicht zu vergessen: Von den neuen Möglichkeiten des Lernens müssten am Schluss die Patientinnen und Patienten profitieren.

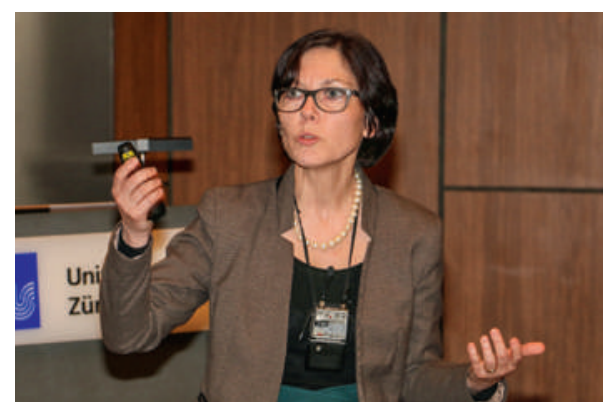

Martina Kadmon, Professorin für Medizinische Ausbildung an der Universität Oldenburg.

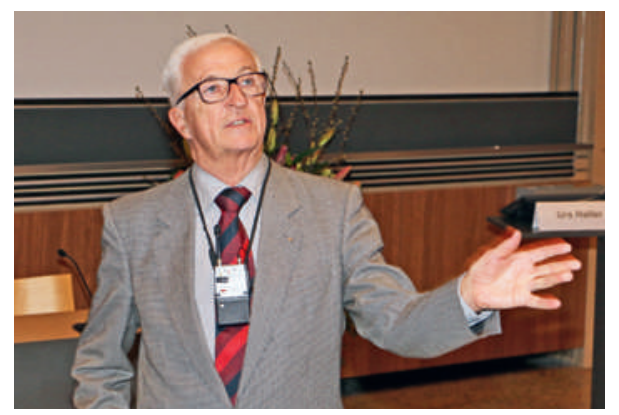

Urs Haller stellte das E-Learning-Portal Egone vor.

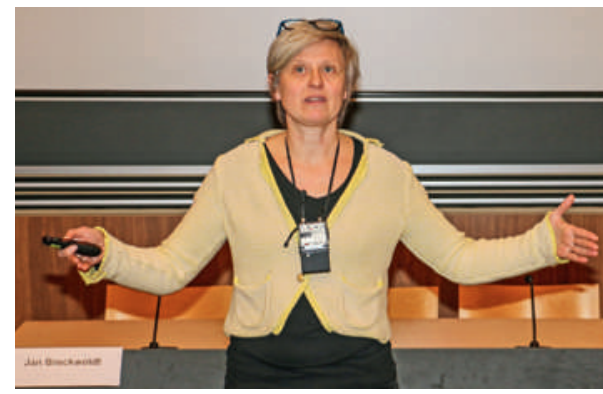

Tanja Krones, Leitende Ärztin Ethik am USZ.

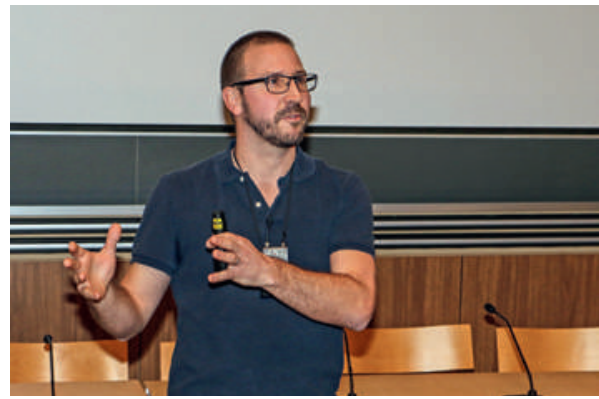

Zunehmend realistischere Simulationen: Adrian Marty bei seinem Referat.

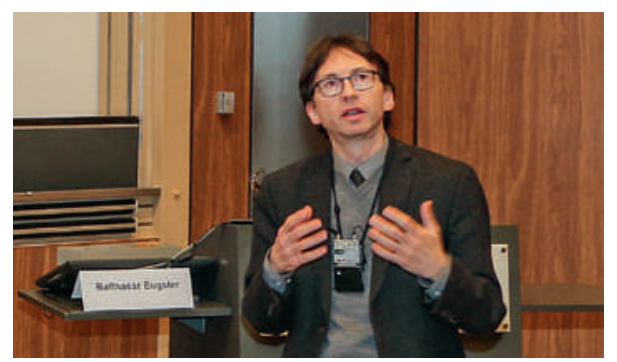

Balthasar Eugster leitete das Schlusspodium. 